\title{
Análisis de la diversidad de peces en ambientes asociados al río Paraná en el área de la represa de Yacyretá
}

\author{
Scipioni, N. ${ }^{1}$; Casciotta, J.R. ${ }^{2,3}$; Almirón, A.E. ${ }^{2}$; Santinón, J.J. ${ }^{1}$, Ruiz-Díaz, F.J. ${ }^{1}$ \\ ${ }^{1}$ Univ. Nac. Nordeste, Fac.Cs.Vet., Inst.Ictiología, Sargento Cabral 2139, Corrientes (3400), Argentina. \\ ${ }^{2}$ Univ. Nac. La Plata, Fac. Cs. Nat. (La Plata, Argentina). ${ }^{3}$ Com. Invest. Científ. CIC (La Plata, Argentina). \\ E-mailnicolas-scipioni@hotmail.com
}

\begin{abstract}
Resumen
Scipioni, N.; Casciotta, J.R.; Almirón, A.E.; Santinón, J.J.; Ruiz-Díaz, F.J.: Análisis de la diversidad de peces en ambientes asociados al río Paraná en el área de la represa de Yacyretá. Rev. vet. 27: 2, 86-92, 2016. El objetivo de este trabajo fue evaluar la diversidad de la ictiofauna en el valle aluvial del río Paraná, entre las localidades de Ituzaingó y Puerto Abra, zona de influencia de la represa de Yacyretá (Argentina). Los datos incluyen el período 2004-2015. Las campañas se realizaron cada tres meses (cuatro por año), abarcando así todas las estaciones. Los peces fueron colectados empleando un equipo de pesca eléctrica. Se eligieron cuatro zonas de muestreos representativas del gradiente longitudinal del río. La diversidad alfa fue medida a través de los índices de Shannon-Wiener, el índice de diversidad verdadera de Jost, el índice de diversidad de Simpson y el índice de dominancia de Simpson, mientras que la diversidad beta se evaluó con el índice de Whittaker y el coeficiente de Jaccard. Además se realizó un análisis de correlación entre los valores de diversidad y la distancia de las zonas con respecto a la represa. Se identificaron 173 especies de peces distribuidas en 11 órdenes y 32 familias. La riqueza estimada para este tramo del río fue de 200 especies. La diversidad específica de las diferentes zonas, sus curvas de rango abundancia y las curvas de acumulación de especies tendieron a disminuir aguas abajo de la represa. Los índices de diversidad alfa utilizados arrojaron los valores más altos en la zona de Itá Ibaté, de posición intermedia a la represa. La diversidad beta fue moderada y similar entre ambientes. Los resultados sugieren diferencias en la estructura de la comunidad de peces en los ambientes del valle aluvial estudiado, que responden a cambios ambientales relacionados con el gradiente longitudinal desde Ituzaingó como punto más cercano a la represa, hasta Puerto Abra como punto más distante.
\end{abstract}

Palabras clave: peces, diversidad específica, río Paraná, represa Yaciretá, Argentina.

\begin{abstract}
Scipioni, N.; Casciotta, J.R.; Almirón, A.E.; Santinón, J.J.; Ruiz-Díaz, F.J.: Analysis of fish diversity in environments associated to the Parana River in the area of the Yacyretá dam. Rev. vet. 27: 2, 86-92, 2016. The diversity of the fish fauna in the alluvial valley of the Paraná River, between the cities of Ituzaingó and Puerto Abra, area of influence of the Yacyretá dam, was evaluated. Data used range from 2004 to 2015. Campaigns are conducted every three months (four per year), covering all seasons. Fish were collected using electrofishing equipment. Four representative zones of the longitudinal gradient of the river were chosen. alpha diversity was measured by the Shannon-Wiener index, the true diversity index by Jost, the Simpson diversity index, and Simpson dominance index, while the beta diversity was assessed with the Whittaker index and Jaccard coefficient. A correlation analysis between the values of diversity and distance from the dam, was also performed. A total of 173 fish species, distributed in 11 orders and 32 families, were identified. Species diversity of different areas, their abundance range curves and curves of accumulation of species, tend to decrease downstream of the dam. Alfa diversity indices used show the highest values in the area of Itá Ibaté, halfway position to the dam. Beta diversity was moderate and similar between environments. The results suggest differences in the structure of the fish community in floodplain environments that respond to environmental changes related to the longitudinal gradient from Ituzaingó as closer to the dam point to Puerto Abra as the most distant point.
\end{abstract}

Key words: fish, species diversity, Paraná River, Yacyretá dam, Argentina. 


\section{INTRODUCCIÓN}

El río Paraná posee la segunda mayor cuenca de América del Sur y es el principal río de la cuenca del Plata, extendiéndose $3.780 \mathrm{~km}$ desde la confluencia de los ríos Paranaíba y Grande (Brasil) hasta el estuario de La Plata (Argentina). Comprende la sección denominada Paraná superior, desde la confluencia del río Grande hasta las cataratas de Sete Quedas en el estado de Guairá, Brasil, la sección llamada Paraná medio que se extiende desde Sete Quedas hasta los saltos de YacyretáApipé y por último la sección del Paraná Inferior, desde los saltos de Yacyretá-Apipé hasta el Delta ${ }^{2,4}$.

Sobre el territorio de la Provincia de Corrientes es un río de valle amplio que se abre en varios brazos que encierran grandes islas. Tras la represa de Yacyretá el río presenta una clara dirección hacia el oeste, ensanchándose progresivamente y ramificándose en varios canales que forman gran cantidad de islas fluviales.

Los ríos con valle de inundación están entre los ecosistemas más productivos y diversos del mundo, están dominados por perturbaciones y caracterizados por altos niveles de diversidad de hábitat, además de una biota adaptada para explotar la heterogeneidad espacio-temporal ${ }^{27,29}$.

El valle aluvial es una parte integral del ecosistema fluvial, donde el régimen de inundación y su conectividad con el cauce principal determina en gran medida la dinámica de las comunidades de peces que habitan en estos ambientes ${ }^{1,15}$.

Muchos vertebrados, como los peces que viven en el canal principal, dependen del valle aluvial para abastecerse de alimentos, para el desove y para refugiarse; al mismo tiempo dependen del régimen hidrológico que posibilita el acceso a estos ambientes ${ }^{15}$.

La construcción de represas ha aumentado en los últimos años, constituyendo un proceso tan intenso y dramático que resulta en la creación de nuevos ecosistemas. La ocurrencia de impacto ambiental es inherente a cualquier embalse ${ }^{3,5}$.

La regulación artificial del río altera los regímenes de perturbaciones naturales que mantienen altos niveles de diversidad y conectividad a través del paisaje fluvial, lo que resulta en una pérdida de la heterogeneidad del hábitat y de la biodiversidad. Varias formas de regulación de los ríos, como por ejemplo la construcción de represas, el dragado y los diques artificiales, han perturbado la conectividad entre las unidades ecológicas de la llanura de inundación. Esta regulación reduce la disponibilidad de ambientes para los peces, tanto juveniles como adultos, lo cual conlleva a un declive de sus poblaciones ${ }^{13,28}$.

En la cuenca del río Paraná y sus ambientes asociados, la comunidad de peces está integrada por alrededor de 400 especies ${ }^{20}$. En el río Paraná superior han sido mencionadas 182 especies ${ }^{12}$, pero en los ambientes asociados a él, se registró un número menor que osciló entre 40 a 59 especies ${ }^{10}$.
En Argentina existe un total de 100 especies registradas aguas abajo de la confluencia con el río Paraguay ${ }^{22}$, mientras que en el Parque Nacional Pre-Delta, la información más reciente menciona 185 especies. En este sector, la estructuración espacial provista por los extensos lechos de macrófitas y los cambios del nivel de agua, determinan patrones de diversidad a escala de hábitat en el litoral de lagunas y canales ${ }^{9}$. Estos parches de vegetación proveen refugio y alimento a una gran variedad de especies de pequeños Characiformes y Siluriformes, así como a ejemplares juveniles de especies migradoras y típicas del canal principal ${ }^{23}$.

Los cambios producidos en la composición longitudinal en ensambles de peces es un fenómeno común en ríos templados, asumiendo que la riqueza de especies aumenta aguas abajo, probablemente por un incremento en la diversidad de hábitat ${ }^{11,19,24}$.

El objetivo de este trabajo fue analizar las variaciones en los ensambles de peces a lo largo de un gradiente longitudinal, aguas abajo de la represa Yacyretá, mediante un análisis de la diversidad local (diversidad alfa), así como caracterizar el grado de intercambio de especies entre cuatro zonas del valle aluvial (diversidad beta) y confeccionar curvas de rango-abundancia y de acumulación de especies.

\section{MATERIAL Y MÉTODOS}

Selección de sitios y periodicidad de muestreos. Se seleccionaron cuatro zonas representativas del gradiente ambiental del río Paraná aguas abajo de la represa de Yacyretá, a saber: 1- Ituzaingó (ITU) S $27^{\circ} 35^{\prime} \mathrm{W}$ $56^{\circ} 43^{\prime}$; 2- Itá Ibaté (ITA) S $27^{\circ} 25^{\prime} \mathrm{W} 57^{\circ} 20^{\prime}$; 3-Yahapé (YAH) S $27^{\circ} 22^{\prime}$ W $57^{\circ} 39^{\prime}$ y 4-Puerto Abra (ABR) S $27^{\circ} 19^{\prime} \mathrm{W} 57^{\circ} 52^{\prime}$.

Las zonas de muestreo elegidas poseen ambientes que conforman el valle aluvial del río Paraná. En cada una se seleccionaron tres sitios representativos del valle aluvial, con conexión permanente con el cauce principal que fluctúa respondiendo a los distintos niveles hidrológicos (Tabla 1).

Los datos y muestras se tomaron desde noviembre de 2004 a agosto de 2015. Los muestreos se realizaron en el margen izquierdo del río Paraná en el tramo comprendido entre la represa Yacyretá y la confluencia con el río Paraguay cada 2 meses sumando un total de 4 campañas por año.

Obtención de las muestras de peces. Para la captura de peces se utilizó un equipo de pesca eléctrica marca Smith Root modelo IV A diseñado para aguas con baja conductividad eléctrica, montado en una embarcación de fibra de vidrio y alimentado por un generador de electricidad marca Honda modelo 2.500CX.

El tiempo de cada captura fue aproximadamente de 1.200 segundos que fueron registrados junto con el amperaje de retorno para los cálculos de estandarización. Los peces capturados en el proceso fueron colocados en baldes con agua del lugar y rotulados por ambiente. 
Esta técnica permitió obtener medidas confiables de captura por unidad de esfuerzo (“CPUE”) ${ }^{25}$, así como evitar el sacrificio de la mayor parte de los peces capturados, que fueron devueltos al agua luego de su identificación.

Los ejemplares fueron clasificados al nivel de especies en el campo. Cuando esto no fue posible se conservó una muestra en formol al 10\% para su identificación en el laboratorio. Para cada especie se consideró la abundancia relativa estandarizada a una hora de captura. Con la identificación taxonómica se elaboró una lista de especies.

Análisis de datos. Una vez obtenida la lista de especies y sus abundancias se realizaron los cálculos de rarefacción utilizando el algoritmo de Jacknife2 implementado en el software EstimateS ${ }^{\circledR}$ 9.0. Además se calcularon los porcentajes de cada orden de peces, curvas de rango-abundancia y curvas de acumulación de especies utilizando Excel $2013^{18,21}$.

Diversidad alfa. La diversidad alfa se analizó empleando el índice de abundancia proporcional como el de equitabilidad de Shannon-Wiener $\left(H^{\prime}=-\sum P i \ln \right.$ $P i$; donde: $\mathrm{Pi}=$ proporción de individuos de la especie $i$ respecto al total de individuos), el índice de diversidad verdadera $\left({ }^{1} \mathrm{D}=\exp \left(\mathrm{H}^{\prime}\right)\right.$; donde: ${ }^{1} \mathrm{D}=$ Diversidad verdadera, $\mathrm{H}^{\prime}=$ índice de Shannon-Wiener) ${ }^{14}$, el índice de dominancia de Simpson $\left(\lambda=\sum P i^{2}\right.$; donde: $\mathrm{Pi}=$ proporción de individuos de la especie i respecto al total de individuos) y el índice de diversidad de Simpson (1- $\lambda$; donde: $\lambda=$ índice de dominancia de Simpson).

La significancia estadística fue aceptada en $\mathrm{p}=0,05$; a los valores de diversidad obtenidos se les realizo una prueba T utilizando el software estadístico Infostat ${ }^{8}$.

Diversidad beta. La diversidad beta se analizó empleando el índice de reemplazo de especies de Whittaker $\left(\beta_{w}=S / \alpha-1\right.$; donde: $\mathrm{S}=$ número total de especies recolectadas en el sistema, $\alpha=$ diversidad media de las muestras) y el índice de similitud de Jaccard ( $I j=c /$ $a+b-c$; donde: $\mathrm{c}=$ número de especies presentes en ambos sitios $\mathrm{A}$ y $\mathrm{B}, \mathrm{a}=$ número de especies presentes en el sitio $\mathrm{A}$ y $\mathrm{b}=$ número de especies presentes en el sitio $\mathrm{B}$ ).

Además se realizó un análisis de correlación de Pearson implementado en el software Infostat para determinar si hubo relación entre los valores de diversidad y la distancia de cada zona con respecto a la represa.

\section{RESULTADOS}

\section{Riqueza de especies}

En el tramo comprendido entre la represa de Yacyretá y la confluencia con el río Paraguay, desde noviembre de 2004 a agosto de 2015, se capturaron 266.456 individuos correspondientes a 173 especies de peces, distribuidas en 32 familias y 11 órdenes. La mayoría de las especies eran de pequeño tamaño y no migradoras (Tabla 2).

Tabla 2. Lista de las especies capturadas ( $\mathrm{X}=$ presencia) en los sitios de muestreo: Ituzaingó (A), Itá-Ibaté (B), Yahapé (C), Puerto Abra (D) y Nº de individuos por zona.

\begin{tabular}{lllll}
\hline Especies & A & B & C & D \\
\hline
\end{tabular}

Myliobatiformes

Potamotrygon motoro

Cupleiformes

Lycengraulis grossidens

Characiformes

Hemiodus orthonops

Apareiodon affinis

Curimatella dorsalis

Cyphocharax platanus

Cyphocharax saladensis

Cyphocharax spilotus

Cyphocharax voga

Potamorhina squamoralevis

Psectrogaster curviventris

Steindachnerina brevipinna

Steindachnerina conspersa

Prochilodus lineatus

Leporinus acutidens

Leporinus lacustris

Leporinus obtusidens

Leporinus striatus

Schizodon borellii

Schizodon nasutus

Schizodon platae

Hoplerythrinus unitaeniatus

Hoplias malabaricus

Pyrrhulina australis

Metynnis mola

Myleus tiete

Mylossoma duriventre

Piaractus mesopotamicus

Pygocentrus nattereri

Serrasalmus maculatus
2735'22, 60"S; 5645'9,90”W

27²5'44,70”S; 57²1'39,50”'W

27²6’36,10”'S; 57¹9'12,50”'W

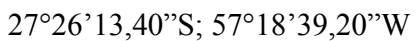

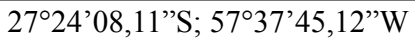

27²3'32,13”'S; 57³8'05,95”'W 27²3'35,24”'S; 57³7'36,37'”W

$27^{\circ} 18^{\prime} 43,70^{\prime \prime} \mathrm{S} ; 57^{\circ} 54^{\prime} 15,40^{\prime \prime} \mathrm{W}$

27¹9'25,46”'S; 5752' 50,66”'W

$27^{\circ} 18^{\prime} 43,20^{\prime \prime}$; 5 5053'12,20”'W 


\begin{tabular}{|c|c|c|c|c|c|c|c|c|c|}
\hline Especies & $\mathbf{A}$ & B & $\mathbf{C}$ & $\mathbf{D}$ & Especies & $\mathbf{A}$ & B & $\mathrm{C}$ & $\mathbf{D}$ \\
\hline Serrasalmus marginatus & $\mathrm{x}$ & $\mathrm{x}$ & $\mathrm{x}$ & $\mathrm{x}$ & $\overline{\text { Trachelyopterus lucenai }}$ & $\mathrm{x}$ & & $\mathrm{x}$ & \\
\hline Acestrorhynchus pantaneiro & $\mathrm{x}$ & $\mathrm{x}$ & $\mathrm{x}$ & $\mathrm{x}$ & Iheringichthys labrosus & $\mathrm{x}$ & $\mathrm{x}$ & $\mathrm{x}$ & \\
\hline Aphyocharax anisitsi & $\mathrm{x}$ & $\mathrm{x}$ & $\mathrm{x}$ & $\mathrm{x}$ & Pimelodus absconditus & $\mathrm{x}$ & & $\mathrm{x}$ & $\mathrm{x}$ \\
\hline Aphyocharax dentatus & $\mathrm{x}$ & $\mathrm{x}$ & $\mathrm{x}$ & $\mathrm{x}$ & Pimelodus albicans & & $\mathrm{x}$ & & \\
\hline Aphyocharax nattereri & $\mathrm{x}$ & $\mathrm{x}$ & $\mathrm{x}$ & $\mathrm{x}$ & Pimelodus argenteus & & $\mathrm{x}$ & $\mathrm{x}$ & $\mathrm{x}$ \\
\hline Aphyocharax rathbuni & $\mathrm{x}$ & $\mathrm{x}$ & $\mathrm{x}$ & $\mathrm{x}$ & Pimelodus maculatus & $\mathrm{x}$ & $\mathrm{x}$ & $\mathrm{x}$ & $\mathrm{x}$ \\
\hline Prionobrama paraguayensis & $\mathrm{x}$ & $\mathrm{x}$ & $\mathrm{x}$ & $\mathrm{x}$ & Pimelodus ornatus & & & $\mathrm{x}$ & \\
\hline Triportheus nematurus & $\mathrm{x}$ & $\mathrm{x}$ & $\mathrm{x}$ & $\mathrm{x}$ & Pseudoplatystoma reticulatum & & & $\mathrm{x}$ & \\
\hline Triportheus pantanensis & & $\mathrm{x}$ & & & Sorubim lima & $\mathrm{x}$ & & & \\
\hline Charax leticiae & $\mathrm{x}$ & $\mathrm{x}$ & $\mathrm{x}$ & $\mathrm{x}$ & Microglanis cottoides & & $\mathrm{x}$ & $\mathrm{x}$ & \\
\hline Charax stenopterus & & $\mathrm{x}$ & $\mathrm{x}$ & $\mathrm{x}$ & Pseudopimelodus mangurus & & $\mathrm{x}$ & & \\
\hline Cynopotamus argenteus & & $\mathrm{x}$ & & $\mathrm{x}$ & Imparfinis mishky & $\mathrm{x}$ & & & \\
\hline Cynopotamus kincaidi & $\mathrm{x}$ & & & $\mathrm{x}$ & Phenacorhamdia tenebrosa & $\mathrm{x}$ & & & \\
\hline Galeocharax humeralis & & & & $\mathrm{x}$ & Pimelodella gracilis & $\mathrm{x}$ & $\mathrm{x}$ & $\mathrm{x}$ & $\mathrm{x}$ \\
\hline Phenacogaster tegatus & $\mathrm{x}$ & $\mathrm{x}$ & $\mathrm{x}$ & $\mathrm{x}$ & Pimelodella laticeps & $\mathrm{x}$ & $\mathrm{x}$ & $\mathrm{x}$ & $\mathrm{x}$ \\
\hline Roeboides affinis & $\mathrm{x}$ & $\mathrm{x}$ & & $\mathrm{x}$ & Pimelodella taenioptera & $\mathrm{x}$ & $\mathrm{x}$ & $\mathrm{x}$ & $\mathrm{x}$ \\
\hline Roeboides descalvadensis & $\mathrm{x}$ & $\mathrm{x}$ & $\mathrm{x}$ & $\mathrm{x}$ & Rhamdia quelen & $\mathrm{x}$ & $\mathrm{x}$ & $\mathrm{x}$ & $\mathrm{x}$ \\
\hline Roeboides microlepis & & $\mathrm{x}$ & $\mathrm{x}$ & & Bunocephalus doriae & $\mathrm{x}$ & $\mathrm{x}$ & $\mathrm{x}$ & $\mathrm{x}$ \\
\hline Heterocheirodon yatai & & & $\mathrm{x}$ & & Pseudobunocephalus iheringii & & & & $\mathrm{x}$ \\
\hline Odontostilbe paraguayensis & $\mathrm{x}$ & $\mathrm{x}$ & $\mathrm{x}$ & $\mathrm{x}$ & Ochmacanthus batrachostomus & & $\mathrm{x}$ & & $\mathrm{x}$ \\
\hline Odontostilbe pequira & $\mathrm{x}$ & $\mathrm{x}$ & $\mathrm{x}$ & $\mathrm{x}$ & Ituglanis eichorniarum & & $\mathrm{x}$ & $\mathrm{x}$ & \\
\hline Serrapinnus calliurus & $\mathrm{x}$ & $\mathrm{x}$ & $\mathrm{x}$ & $\mathrm{x}$ & Callichthys callichthys & & & $\mathrm{x}$ & \\
\hline Serrapinnus kriegi & $\mathrm{x}$ & $\mathrm{x}$ & $\mathrm{x}$ & $\mathrm{x}$ & Hoplosternum littorale & & $\mathrm{x}$ & & \\
\hline Salminus brasiliensis & $\mathrm{x}$ & $\mathrm{x}$ & $\mathrm{x}$ & $\mathrm{x}$ & Lepthoplosternum pectorale & $\mathrm{x}$ & $\mathrm{x}$ & & $\mathrm{x}$ \\
\hline Piabucus melanostomus & $\mathrm{x}$ & $\mathrm{x}$ & $\mathrm{x}$ & $\mathrm{x}$ & Corydoras aeneus & & & & $\mathrm{x}$ \\
\hline Gymnocorymbus ternetzi & $\mathrm{x}$ & $\mathrm{x}$ & $\mathrm{x}$ & $\mathrm{x}$ & Corydoras hastatus & $\mathrm{x}$ & $\mathrm{x}$ & $\mathrm{x}$ & $\mathrm{x}$ \\
\hline Poptella paraguayensis & $\mathrm{x}$ & $\mathrm{x}$ & $\mathrm{x}$ & $\mathrm{x}$ & Corydoras paleatus & & $\mathrm{x}$ & & $\mathrm{x}$ \\
\hline Bryconamericus exodon & $\mathrm{x}$ & $\mathrm{x}$ & $\mathrm{x}$ & $\mathrm{x}$ & Corydoras polystictus & & & & $\mathrm{x}$ \\
\hline Bryconamericus iheringii & $\mathrm{x}$ & $\mathrm{x}$ & & & Hypoptopoma inexspectatum & $\mathrm{x}$ & $\mathrm{x}$ & $\mathrm{x}$ & $\mathrm{x}$ \\
\hline Tetragonopterus argenteus & $\mathrm{x}$ & $\mathrm{x}$ & $\mathrm{x}$ & $\mathrm{x}$ & Otocinclus vestitus & $\mathrm{x}$ & $\mathrm{x}$ & $\mathrm{x}$ & $\mathrm{x}$ \\
\hline Astyanax abramis & $\mathrm{x}$ & $\mathrm{x}$ & $\mathrm{x}$ & $\mathrm{x}$ & Hisonotus maculipinnis & $\mathrm{x}$ & $\mathrm{x}$ & $\mathrm{x}$ & $\mathrm{x}$ \\
\hline Astyanax asuncionensis & $\mathrm{x}$ & $\mathrm{x}$ & $\mathrm{x}$ & $\mathrm{x}$ & Ancistrus cirrhosus & & $\mathrm{x}$ & $\mathrm{x}$ & \\
\hline Astyanax correntinus & $\mathrm{x}$ & $\mathrm{x}$ & $\mathrm{x}$ & & Hypostomus arecuta & & & $\mathrm{x}$ & \\
\hline Astyanax erythropterus & $\mathrm{x}$ & $\mathrm{x}$ & & & Hypostomus boulengeri & & $\mathrm{x}$ & & \\
\hline Astyanax pynandi & $\mathrm{x}$ & & & & Hypostomus cochliodon & & & $\mathrm{x}$ & \\
\hline Astyanax rutilus & $\mathrm{x}$ & $\mathrm{x}$ & $\mathrm{x}$ & $\mathrm{x}$ & Hypostomus commersoni & $\mathrm{x}$ & $\mathrm{x}$ & $\mathrm{x}$ & \\
\hline Markiana nigripinnis & & & $\mathrm{x}$ & & Hypostomus latifrons & & $\mathrm{x}$ & & \\
\hline Psellogrammus kennedyi & $\mathrm{x}$ & $\mathrm{x}$ & $\mathrm{x}$ & $\mathrm{x}$ & Hypostomus regani & $\mathrm{x}$ & $\mathrm{x}$ & $\mathrm{x}$ & \\
\hline Hemigrammus mahnerti & $\mathrm{x}$ & $\mathrm{x}$ & $\mathrm{x}$ & $\mathrm{x}$ & Hypostomus ternetzi & & $\mathrm{x}$ & $\mathrm{x}$ & \\
\hline Hemigrammus ulreyi & $\mathrm{x}$ & $\mathrm{x}$ & $\mathrm{x}$ & $\mathrm{x}$ & Hypostomus uruguayensis & & & $\mathrm{x}$ & \\
\hline Hyphessobrycon anisitsi & $\mathrm{x}$ & $\mathrm{x}$ & & $\mathrm{x}$ & Pterygoplichthys ambrosettii & $\mathrm{x}$ & $\mathrm{x}$ & $\mathrm{x}$ & $\mathrm{x}$ \\
\hline Hyphessobrycon elachys & $\mathrm{x}$ & $\mathrm{x}$ & $\mathrm{x}$ & $\mathrm{x}$ & Rhinelepis strigosa & & $\mathrm{x}$ & & \\
\hline Hyphessobrycon eques & $\mathrm{x}$ & $\mathrm{x}$ & $\mathrm{x}$ & $\mathrm{x}$ & Farlowella hahni & $\mathrm{x}$ & $\mathrm{x}$ & $\mathrm{x}$ & $\mathrm{x}$ \\
\hline Hyphessobrycon igneus & & $\mathrm{x}$ & & $\mathrm{x}$ & Loricaria apeltogaster & & $\mathrm{x}$ & & \\
\hline Hyphessobrycon luetkenii & $\mathrm{x}$ & $\mathrm{x}$ & $\mathrm{x}$ & $\mathrm{x}$ & Loricaria luciae & $\mathrm{x}$ & $\mathrm{x}$ & $\mathrm{x}$ & $\mathrm{x}$ \\
\hline Hyphessobrycon meridionalis & & & & $\mathrm{x}$ & Loricaria simillima & & $\mathrm{x}$ & $\mathrm{x}$ & $\mathrm{x}$ \\
\hline Hyphessobrycon wajat & $\mathrm{x}$ & $\mathrm{x}$ & $\mathrm{x}$ & & Loricariichthys anus & & & & $\mathrm{x}$ \\
\hline Moenkhausia dichroura & $\mathrm{x}$ & $\mathrm{x}$ & $\mathrm{x}$ & $\mathrm{x}$ & Loricariichthys edentatus & $\mathrm{x}$ & $\mathrm{x}$ & $\mathrm{x}$ & \\
\hline Moenkhausia intermedia & $\mathrm{x}$ & $\mathrm{x}$ & $\mathrm{x}$ & $\mathrm{x}$ & Loricariichthys labialis & $\mathrm{x}$ & $\mathrm{x}$ & $\mathrm{x}$ & $\mathrm{x}$ \\
\hline Moenkhausia sanctaefilomenae & $\mathrm{x}$ & $\mathrm{x}$ & $\mathrm{x}$ & $\mathrm{x}$ & Loricariichthys melanocheilus & $\mathrm{x}$ & $\mathrm{x}$ & & $\mathrm{x}$ \\
\hline Characidium cf. Zebra & $\mathrm{x}$ & $\mathrm{x}$ & $\mathrm{x}$ & $\mathrm{x}$ & Loricariichthys platymetopon & $\mathrm{x}$ & $\mathrm{x}$ & $\mathrm{x}$ & $\mathrm{x}$ \\
\hline Characidium rachovii & $\mathrm{x}$ & & & $\mathrm{x}$ & Loricariichthys rostratus & & $\mathrm{x}$ & & \\
\hline Siluriformes & & & & & Paraloricaria vetula & & & & $\mathrm{x}$ \\
\hline Ossancora eigenmanni & & $\mathrm{x}$ & $\mathrm{x}$ & & Rineloricaria lanceolata & $\mathrm{x}$ & $\mathrm{x}$ & & $\mathrm{x}$ \\
\hline Platydoras armatulus & $\mathrm{x}$ & $\mathrm{x}$ & $\mathrm{x}$ & $\mathrm{x}$ & Rineloricaria parva & $\mathrm{x}$ & $\mathrm{x}$ & $\mathrm{x}$ & $\mathrm{x}$ \\
\hline Pterodoras granulosus & & & $\mathrm{x}$ & & Rineloricaria misionera & & & & $\mathrm{x}$ \\
\hline Ageneiosus inermis & & & $\mathrm{x}$ & & Gymnotiformes & & & & \\
\hline Trachelyopterus aff. albicrux & $\mathrm{x}$ & $\mathrm{x}$ & $\mathrm{x}$ & $\mathrm{x}$ & Eigenmannia trilineata & & $\mathrm{x}$ & $\mathrm{x}$ & $\mathrm{x}$ \\
\hline
\end{tabular}




\begin{tabular}{|c|c|c|c|c|}
\hline Especies & $\mathbf{A}$ & B & $\mathrm{C}$ & D \\
\hline Sternopygus macrurus & & & $\mathrm{x}$ & \\
\hline Apteronotus brasiliensis & $\mathrm{x}$ & & $\mathrm{x}$ & \\
\hline Ramphichthys hahni & & & $\mathrm{x}$ & \\
\hline Gymnorhamphichthys britskii & & $\mathrm{x}$ & & $\mathrm{x}$ \\
\hline Brachyhypopomus bombilla & & $\mathrm{x}$ & & \\
\hline Brachyhyроротиs draco & $\mathrm{x}$ & $\mathrm{x}$ & $\mathrm{x}$ & \\
\hline Brachyhypopomus gauderio & & $\mathrm{x}$ & & \\
\hline Gymnotus inaequilabiatus & $\mathrm{x}$ & $\mathrm{x}$ & $\mathrm{x}$ & \\
\hline \multicolumn{5}{|l|}{ Atheriniformes } \\
\hline Odontesthes bonariensis & & $\mathrm{x}$ & & \\
\hline Odontesthes perugiae & & & $\mathrm{x}$ & \\
\hline \multicolumn{5}{|l|}{ Cyprinodontiformes } \\
\hline Melanorivulus punctatus & & & $\mathrm{x}$ & \\
\hline \multicolumn{5}{|l|}{ Beloniformes } \\
\hline Potamorrhaphis eigenmanni & $\mathrm{x}$ & $\mathrm{x}$ & $\mathrm{x}$ & $\mathrm{x}$ \\
\hline Pseudotylosurus angusticeps & $\mathrm{x}$ & $\mathrm{x}$ & $\mathrm{x}$ & \\
\hline \multicolumn{5}{|l|}{ Synbranchiformes } \\
\hline Synbranchus marmoratus & $\mathrm{x}$ & $\mathrm{x}$ & $\mathrm{x}$ & $\mathrm{x}$ \\
\hline \multicolumn{5}{|l|}{ Perciformes } \\
\hline Pachyurus bonariensis & $\mathrm{x}$ & $\mathrm{x}$ & $\mathrm{x}$ & $\mathrm{x}$ \\
\hline Plagioscion ternetzi & & & $\mathrm{x}$ & $\mathrm{x}$ \\
\hline Bujurquina vittata & & $\mathrm{x}$ & $\mathrm{x}$ & $\mathrm{x}$ \\
\hline Cichlasoma dimerus & $\mathrm{x}$ & $\mathrm{x}$ & $\mathrm{x}$ & $\mathrm{x}$ \\
\hline Laetacara dorsigera & $\mathrm{x}$ & $\mathrm{x}$ & $\mathrm{x}$ & $\mathrm{x}$ \\
\hline Apistogramma borellii & $\mathrm{x}$ & $\mathrm{x}$ & $\mathrm{x}$ & $\mathrm{x}$ \\
\hline Apistogramma commbrae & $\mathrm{x}$ & $\mathrm{x}$ & $\mathrm{x}$ & $\mathrm{x}$ \\
\hline Apistogramma trifasciata & & & $\mathrm{x}$ & $\mathrm{x}$ \\
\hline Crenicichla jupiaensis & & & $\mathrm{x}$ & \\
\hline Crenicichla lepidota & $\mathrm{x}$ & $\mathrm{x}$ & $\mathrm{x}$ & $\mathrm{x}$ \\
\hline Crenicichla semifasciata & $\mathrm{x}$ & $\mathrm{x}$ & $\mathrm{x}$ & $\mathrm{x}$ \\
\hline Crenicichla taikyra & $\mathrm{x}$ & & $\mathrm{x}$ & \\
\hline Crenicichla vittata & $\mathrm{x}$ & $\mathrm{x}$ & $\mathrm{x}$ & $\mathrm{x}$ \\
\hline Gymnogeophagus balzanii & $\mathrm{x}$ & $\mathrm{x}$ & $\mathrm{x}$ & $\mathrm{x}$ \\
\hline \multicolumn{5}{|l|}{ Pleuronectiformes } \\
\hline Catathyridium jenynsii & $\mathrm{x}$ & $\mathrm{x}$ & $\mathrm{x}$ & $\mathrm{x}$ \\
\hline $\mathbf{N}^{0}$ de individuos & 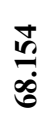 & $\begin{array}{l}\text { ڤे } \\
\text { ले } \\
\text { हे }\end{array}$ & $\begin{array}{l}\mathscr{\infty} \\
\dot{\infty}\end{array}$ & $\begin{array}{l}\vec{J} \\
\infty \\
\dot{b}\end{array}$ \\
\hline
\end{tabular}

Los órdenes con mas abundancia de especies presentes en el valle aluvial de este tramo del río Paraná fueron los Characiformes con 80 especies capturadas (46\%), Siluriformes con 61 especies capturadas $(36 \%)$, Perciformes con 14 especies (8\%), Gymnotiformes con 9 especies $(5 \%)$ y los restantes siete ordenes en total suman 9 especies capturadas, que constituyen el 5\% restante.

La estructura de la comunidad en función de la abundancia proporcional de las especies encontradas describe un modelo de distribución log-normal en todas las zonas (Figura 1).

En las cuatro zonas, la especie más abundante fue $O$. pequira. En Ituzaingó, esta especie representó el $76,9 \%$ del total de individuos capturados, seguida por Moenkhausia intermedia, que constituyó el 6,74\% del total de individuos de esta zona.

En Itá Ibaté $O$. pequira representó el 41,9\%, seguida de $A$. asuncionensis representando el 7,2\% del total capturado para la zona.
En Yahapé $O$. pequira representó el 29,8\% del total capturado, seguida de $A$. asuncionensis que constituyó el 8,5\% del total capturado.

En Puerto Abra, O. pequira representó el 54,7\% del total capturado, seguida de Otocinclus vestitus que constituyó el $15 \%$ del total capturado.

El número total de especies a encontrar, estimado mediante el algoritmo Jacknife2, para este tramo del río, fue de 200 especies según la representación de estos datos en la curva de acumulación de especies (Figura 2). La completitud del muestreo fue de $86 \%$, obteniendo un número de especies muy próximo al estimado.

\section{Diversidad alfa}

Los resultados de los índices utilizados para analizar diversidad alfa mostraron la misma tendencia; es decir, los valores de diversidad fueron menores junto a la represa y luego de su máximo valor en Itá Ibaté, disminuyeron aguas abajo.

El índice de Shannon-Wiener (H') y el índice de diversidad de Simpson resultaron significativamente distintos $(\mathrm{p}<0,05)$, arrojando los valores más altos para Itá Ibaté $\left(H^{\prime}=2,64\right.$ bits; $\left.1-\lambda=0,80\right)$ y los más bajos para Ituzaingó $\left(H^{\prime}=1,83\right.$ bits; $\left.1-\lambda=0,59\right)$. Un comportamiento similar tuvo el índice de diversidad verdadera ( $\left.{ }^{1} \mathrm{D}\right)$. El índice de dominancia de Simpson fue más alto para Ituzaingó $(\lambda=0,41)$ y el más bajo se observó en Itá Ibaté $(\lambda=0,2)$ (Tabla 3$)$.

Tabla 3. Análisis de diversidad específica en el valle aluvial del río Paraná.

\begin{tabular}{lcccccc}
\hline zona & $\mathrm{S}$ & $\mathrm{E}$ & $\mathrm{H}^{\prime}$ & ${ }^{\mathrm{I}} \mathrm{D}$ & $\lambda$ & $1-\lambda$ \\
\hline Ituzaingó & 114 & 4 & 1,83 & 6,23 & 0,41 & 0,59 \\
Itá Ibaté & 135 & 11 & 2,64 & 14,01 & 0,20 & 0,80 \\
Yahapé & 132 & 15 & 2,40 & 11,02 & 0,23 & 0,77 \\
P. Abra & 120 & 8 & 2,40 & 11,02 & 0,22 & 0,78 \\
\hline
\end{tabular}

$\mathrm{S}=$ número total de especies, $\mathrm{E}=$ número de especies exclusivas por zona, $\mathrm{H}^{\prime}$ = índice de Shannon, ${ }^{1} \mathrm{D}=$ índice de Jost, $\lambda=$ índice de dominancia de Simpson, $1-\lambda=$ diversidad de Simpson.

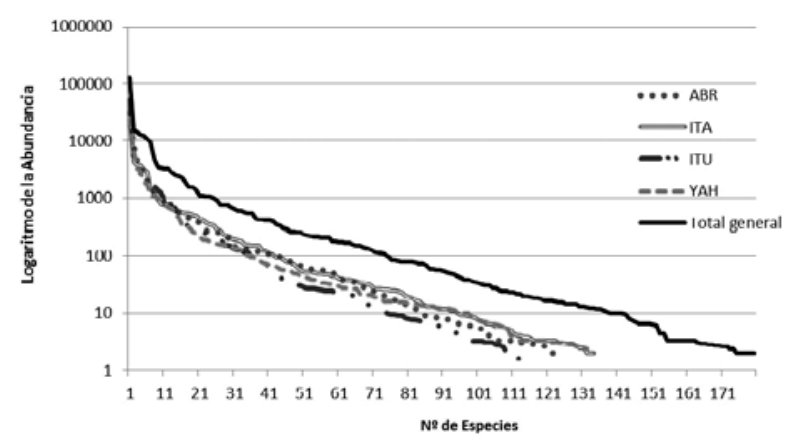

Figura 1. Curvas de rango abundancia para las cuatro zonas y total general. ABR: Puerto Abra, ITA: ItáIbaté, ITU: Ituzaingó, YAH: Yahapé. 


\section{Diversidad beta}

Los valores obtenidos en el análisis de la diversidad beta entre zonas fueron semejantes utilizando los índices de Whittaker y de similitud de Jaccard. El índice de Whittaker osciló entre un máximo de 1,09 para Itá Ibaté y un mínimo de 0,92 para Ituzaingó, mientras que en Yahapé y Puerto Abra resultó de 1,06 y 0,97 respectivamente. El índice de similitud de Jaccard presentó el valor máximo de 0,70 entre Ituzaingó-Itá Ibaté y entre Itá Ibaté-Puerto Abra, mientras que el valor mínimo fue de 0,62 entre Yahapé y Puerto Abra (Tabla 4).

Tabla 4. Índice de similitud de Jaccard (Ij).

\begin{tabular}{lcccc}
\hline zona & Ituzaingó & Itá Ibaté & Yahapé & P. Abra \\
\hline Ituzaingó & $114(4)$ & 0,70 & 0,67 & 0,69 \\
Itá Ibaté & 0,70 & $135(11)$ & 0,69 & 0,70 \\
Yahapé & 0,67 & 0,69 & $132(15)$ & 0,62 \\
P. Abra & 0,69 & 0,70 & 0,62 & $120(8)$ \\
\hline
\end{tabular}

En diagonal: número total de especies y entre paréntesis: número de especies exclusivas.

El análisis de correlación de Pearson mostró una relación positiva de 0,74 entre el índice de ShannonWiener y la distancia de cada zona con respecto a la represa.

\section{DISCUSIÓN}

El río Paraná sustenta una gran diversidad de peces, sin embargo poco se conoce sobre la composición de las especies en el tramo comprendido entre la represa de Yacyretá y la confluencia con el río Paraguay.

Los porcentajes registrados de Characiformes y Siluriformes representan, los dos juntos, el $82 \%$ de todas las especies de peces presentes en el tramo del río estudiado. Estos valores siguen la tendencia normal para la cuenca del neotrópico donde los Characiformes y Siluriformes -entre ambos- constituyen más del $85 \%$ de todas las especies de peces ${ }^{17}$.

En el tramo estudiado se registraron 173 especies de peces, datos que se complementan con los de otros

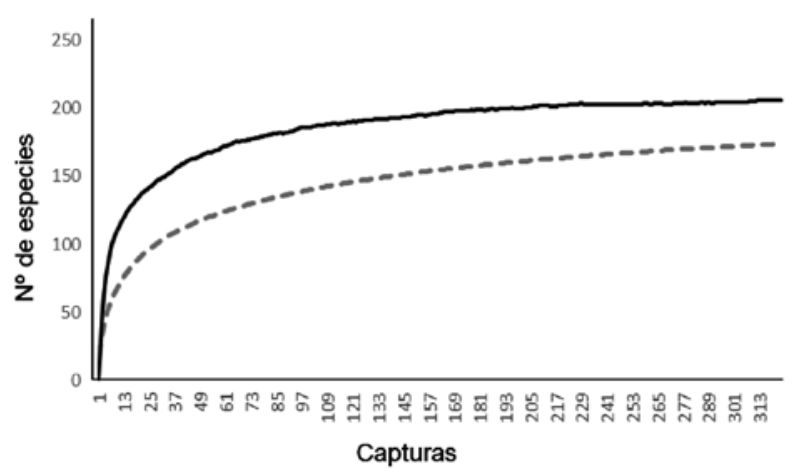

Figura 2. Curva de acumulación de especies. Línea discontinua: riqueza observada; línea continua: riqueza estimada. autores que estudiaron zonas cercanas. Según las estimaciones aquí elaboradas, se considera que las especies faltantes (aproximadamente 27), podrían ser el resultado de varios factores: la selectividad del equipo de pesca eléctrica para capturar determinadas especies; las rayas del género Potamotrygon aunque observadas, no pudieron ser capturadas con esta metodología; la falta de pescas nocturnas por los riesgos en el uso de pesca eléctrica en este tipo de muestreos, las capturas para este trabajo se realizaron durante el día con lo cual muchas especies de Siluriformes y Gymnotiformes, activos durante la noche, no fueron capturados.

En cuanto a la dominancia de especies, en las cuatro zonas de estudio, $O$. pequira resultó el más abundante, particularmente en Ituzaingó, lo que se ve reflejado en el alto valor obtenido según el índice de dominancia Simpson que favorece a las especies dominantes.

Otras especies dominantes en las cuatro zonas fueron $M$. intermedia, $O$. vestitus, $A$. asuncionensis y $P$. paraguayensis. La literatura menciona la dominancia de especies ecológicamente similares; éstas corresponden a individuos de pequeño porte, no migradoras, consideradas eficientes en el uso de los recursos y altamente adaptadas a perturbaciones 7,9,10. La elevada abundancia de una especie y su frecuencia de ocurrencia indicarían la existencia de una perturbación o de otro condicionante en la repartición de recursos, que podría corresponder a una variación diaria del nivel hidrológico a causa del funcionamiento de la represa.

La diversidad específica es uno de los atributos más relevantes de las comunidades, relacionado a su organización y estabilidad ${ }^{16}$ Abundan trabajos relacionando la diversidad íctica con períodos hidrológicos contrastantes, pero los estudios que relacionan los cambios de diversidad con la distancia a embalses son escasos. Los resultados aquí obtenidos muestran que existe una alta correlación entre los valores de diversidad y la distancia de las zonas con respecto a la represa, siendo uno de los posibles factores determinantes de la diversidad en estos ambientes.

En Ituzaingó se registró la diversidad más baja, probablemente debido al menor número de especies halladas y a la alta dominancia de algunas de ellas. Por otra parte, el mayor número de especies, la baja dominancia y el mayor valor de diversidad alfa se registraron en Itá Ibaté, que es una zona de posición intermedia. Por último, los valores de riqueza de especies, dominancia y diversidad alfa volvieron a disminuir en las zonas más alejadas a la represa. Al ser Itá Ibaté un punto de distancia intermedia a la represa de Yacyretá y por presentar los mayores valores de diversidad, puede considerarse que los resultados del presente trabajo coinciden con los postulados de la teoría ecológica de la perturbación intermedia, donde un grado intermedio de perturbación puede permitir la coexistencia de un mayor número de especies ${ }^{6,26}$.

Aún considerando que las zonas estudiadas poseen entre sí una conexión permanente (en mayor o menor grado) y que la distancia entre ellas y con el canal prin- 
cipal es corta, los cálculos de la diversidad beta evidencian que existe un gradiente ambiental determinando los reemplazos de especies entre zonas. Tal variación sería explicada por las particularidades ecológicas de las zonas, los hábitos de vida de sus especies exclusivas y sus abundancias, no tomadas en cuenta por los índices utilizados.

Se concluye que las diferencias en la estructura de la comunidad de peces en los ambientes asociados del valle aluvial estudiado, responden a cambios ambientales relacionados con el gradiente longitudinal desde Ituzaingó como punto más cercano a la represa, hasta Puerto Abra como punto más distante.

Agradecimientos. A la Entidad Binacional Yacyretá (EBY), que a través de su Departamento de Medio Ambiente posibilitó la mayoría de las campañas realizadas. Al personal del Instituto de Ictiología por colaborar en las campañas. A la SGCYT-UNNE por financiar el proyecto (B009-2014). A los establecimientos particulares que permitieron el acceso a los puntos de muestreo.

\section{REFERENCIAS}

1. Agostinho AA, Vazzoler AB, Gomes LC, Okada BK. 1993. Estratificação especial y comportamento de Prochilodus scrofa en distintas fases del ciclo de vida, en la planicie de inundação del alto rio Paraná y embalses de Itaipu, Paraná, Brasil. Rev Hydrobiol Trop 26: 79-90.

2. Agostinho AA, Gomes LC, Zalewski M. 2001. The importance of floodplains for the dynamics of fish communities of the upper river Paraná. Int J Ecohydrol \& Hydrobiol 1: 209-217.

3. Agostinho AA, Pelicice FM, Gomes LC. 2008. Dams and the fish fauna of the neotropical region: impacts and management related to diversity and fisheries. Braz J Biol 68: 1119-1132.

4. Albert JS, Carvalho TP. 2011. Neogene assembly of modern faunas. In: Historical biogeography of neotropical freshwater fishes (Albert JS, Reis RE. eds.), University of California Press, California (USA), p. 119-136.

5. Baxter RM. 1977. Environmental effects of dams and impoundments. Ann Rev Ecol Syst 8: 255-283.

6. Connell JH. 1978. Diversity in tropical rain forest and coral reefs. Science 199: 1302-1310.

7. Delariva RL, Agostinho AA, Nakatani K, Baumgartner G. 1994. Ichthyofauna associated to aquatic macrophytes in the upper Parana River floodplain. Rev Unimar 16: 41-60.

8. Di Rienzo JA, Casanoves F, Balzarini MG, Gonzalez L, Tablada M, Robledo CW. 2015. InfoStat versión 2015. Grupo InfoStat, FCA, Universidad Nacional de Córdoba, Argentina. URL http://www.infostat.com.ar

9. Dibble ED, Pelicice FM. 2010. Influence of aquatic plantspecific habitat on an assemblage of small neotropical floodplain fishes. Ecol Freshwat Fish 19: 381-389.
10. Gomes LC, Bulla CK, Agostinho AA, Vasconcelos LP, Miranda LE. 2012. Fish assemblage dynamics in a neotropical floodplain relative to aquatic macrophytes and the homogenizing effect of a flood pulse. Hydrobiologia 685: 97-107.

11. Gorman OT, Karr JR. 1978. Habitat structure and stream fish communities. Ecology 59: 507-515.

12. Graça WJ, Pavanelli CS. 2007. Peixes da planicie de inundação do alto rio Paraná e áreas adjacentes, Ed. EDUEM, Maringá (Brazil), p. 241.

13. Harris JH, Gehrke PC. 1997. Fish and rivers in stress: The NSW rivers survey. NSW Fisheries Office of Conservation and the Cooperative Research Centre for Freshwater Ecology, Cronulla, New South Wales, Australia, p.298.

14. Jost L. 2006. Entropy and diversity. Oikos 113: 363-374.

15. Junk WJ, Bayley PB, Sparks RE. 1989. The flood pulse concept in river-floodplain systems. In: Proceedings of the International Large River Symposium (Dodge DP, ed.). Canadian Special Publishers Fisheries and Aquatic Sciences, Ottawa, p. 110-127.

16. Krebs JC. 1986. Ecología, ed. Pirámide, Madrid, p. 782.

17. Lowe-McConnell RH. 1987. Ecological studies in tropical fish communities, Cambridge University Press, UK, p. 382.

18. Magurran A.1988. Ecological diversity and its measurement, Princeton University Press, New Jersey, 179 p.

19. Matthews WJ. 1998. Patterns in freshwater fish ecology. Oikos 80: 583-587.

20. Mirande JM, Koerber S. 2015. Checklist of the freshwater fishes of Argentina (CLOFFAR). Ichthyological Contributions of Peces Criollos 36: 1-68.

21. Moreno CE. 2001. Métodos para medir la biodiversidad, Ed. M\&T, Barcelona, p. 84.

22. Neiff JJ, Poi A, Verón MB. 2009. The role of vegetated areas on fish assemblage of the Paraná River floodplain: effects of different hydrological conditions. Neotrop Ichthyol 7: 39-48.

23. Pelicice FM, Agostinho AA, Thomaz SM. 2005. Fish assemblages associated with Egeria in a tropical reservoir: investigating the effects of plant biomass and diel period. Acta Oecologica 27: 9-16.

24. Petry AC, Schulz UH. 2006. Longitudinal changes and indicator species of the fish fauna in the subtropical Sinos River, Brazil. J Fish Biol 69: 272-290.

25. Reynolds JB. 1996. Electrofishing. In: Fisheries Techniques (Murphy BR, Willis DW eds.), $2^{\text {nd }}$ ed., Publ. American Fisheries Society, Maryland, USA, p. 221-253.

26. Roxburgh SH, Shea K, Wilson JB. 2004. The intermediate disturbance hypothesis: Patch dynamics and mechanisms of species coexistence. Ecology 85: 359-371.

27. Tockner K, Stanford AJ. 2002. Riverine flood plains: present state and future trends. Environ Conservat, p 308330. doi: 10.1017/S037689290200022X.

28. Ward JV, Tockner K, Schiemer F. 1999. Biodiversity of floodplain river ecosystems: ecotones and connectivity. Regulated Rivers: Research \& Management 15: 125-139.

29. Welcomme RL. 1979. The fisheries ecology of Floodplain Rivers, Ed. Longman Group, Essex (UK), p. 317. 